\title{
Validación de un constructo para determinar la calidad de los servicios turísticos
}

Diego Mauricio Calvopiña Andrade ${ }^{1}$

\begin{abstract}
RESUMEN
El presente trabajo de investigación explica la validación de un constructo para determinar la calidad de los servicios turísticos, la cual es considerada como la actividad de mayor incidencia mundial en el ámbito político, económico, social, cultural, ambiental y tecnológico. Esta función dejó de basarse únicamente en la concepción del viaje, como se pensaba en la antigüedad. En la actualidad, se trata cada vez más de una actividad que está orientada a un modelo activo y participativo, que sugiere establecer innovadoras estrategias de convivencia, aprendizaje y disfrute de los viajeros y anfitriones. Para ello resulta indispensable considerar a los servicios y su incidencia en la competitividad y en el desarrollo sostenible. La tendencia actual del turismo es poner mayor énfasis en la calidad de los servicios turísticos por considerarla como parte fundamental de la dimensión turística. Así, surge el siguiente problema: ¿cuáles son las dimensiones del constructo de la calidad de los servicios turísticos (percepciones) que influyen en las expectativas del servicio turístico? Se determinó la validez y la fiabilidad del instrumento, corroborando la pertinencia del instrumento seleccionado y el impacto en los criterios de calidad de los servicios turísticos.
\end{abstract}

Palabras-claves: Constructo; SERVQUAL; calidad; servicios; turísticos.

\section{INTRODUCCIÓN}

Esta investigación tiene soporte en teorías y modelos de calidad del servicio turístico, como el Plan Nacional del Buen Vivir del Ecuador, el Plandetur 2020 y los planes de turismo de la provincia de Chimborazo y Riobamba particularmente. Es evidente la alineación del presente estudio con lo estipulado en los Objetivos del Milenio, los Objetivos del Plan Nacional del Buen Vivir, el Código Orgánico de Ordenamiento Territorial, Autonomía y Descentralización y la gobernanza turística mundial y ecuatoriana, como aporte de incidencia social. Para Pride y Ferrell (1997), "es importante destacar que la calidad es determinante en la percepción del cliente sobre un servicio" (p. 35); además, los autores definen la calidad del servicio "como la percepción que tienen los clientes si un servicio satisface o si excede sus expectativas" (p. 12). A partir de lo expuesto, se han caracterizado dos atributos clave dentro de la percepción de la calidad del servicio. Además, se han adoptado los preceptos de Stanton, Etzel y Walker (2004), Castelluci (2009) y Romani (2017). Así, el primer precepto está basado en la calidad de servicio como valoración por el cliente (no por el gestor de la servucción o vendedor del servicio); mientras que el segundo resulta al comparar y contrastar las expectativas iniciales de los clientes, quienes categorizan y valoran la calidad del servicio recibido, con las percepciones reales. Estas expectativas se basan en modelos mentales adquiridos de la experiencia previa o de la publicidad imperante.

Por su lado, los investigadores Parasuraman, Berry y Zeithaml (1988) asumen otros preceptos, ellos señalan que las expectativas del cliente constituyen el factor decisivo al pretender evaluar la calidad del servicio ofertado con el establecimiento determinado. Esto ocurre porque los clientes miden, comparan y evalúan el servicio ofertado según sus expectativas y el nivel esperado. La percepción global del servicio recibido apunta a que la calidad del servicio sea otorgada como la diferencia o contraste entre el servicio esperado versus el servicio percibido.

1 Magíster en Ingeniería Industrial por la Universidad Nacional Mayor de San Marcos. Docente de la Universidad Nacional de Chimborazo. Chimborazo, Ecuador. E-mail: dcalvopina@unach.edu.ec ORCID: 000-0003-4034-4185.

Google académico: https://scholar.google.es/citations?view_op=new articles\&hl=es\&imq=Diego + Mauricio + Calvopi $\% \mathrm{C} 3 \% \mathrm{~B} 1 \mathrm{a}+$ Andrade. 
Relativo al enfoque de calidad de los servicios, los autores Zeithaml, Bitner y Gremler (2005) arguyen que "la calidad de servicio es un componente primordial de las percepciones del cliente" (p. 7). Por su parte, los estudiosos Olmedo (2014) y Romani (2017) argumentan que es la cuestión la que prevalece en las mentes de los clientes al evaluar el servicio, así: "la calidad del servicio también puede ser fundamental para determinar la satisfacción del cliente" (p. 65). Por otro lado, Peñaloza (2007) explicita que la calidad es relevante, ya que "el fin último del marketing es la satisfacción del cliente y esta es posible solo cuando se proporcionan productos y servicios de calidad, los cuales están íntimamente relacionados, motivo por el que resulta obligatorio centrarse en la valoración de la calidad" (p. 36).

Por otro lado, Parasuraman, Berry y Zeithaml (1988) presentan el modelo SERVQUAL (service of quality), el cual es una herramienta para la investigación que funciona al fijar el grado de satisfacción de los clientes (de una organización orientada a los servicios) a través de la brecha existente entre lo esperado y lo recibido. Lo que plantean estos autores resulta complejo, pues es subjetivo evaluar y cuestionar la calidad en los servicios. Por ello, el acercamiento ocurre de forma distinta entre la diferencia de productos físicos tangibles y la servucción, donde las personas gozan de evidentes elementos físicos a la hora de discriminar y juzgar el grado de calidad de un servicio recibido.

Los investigadores citados anteriormente abordan diversos puntos neurálgicos y críticos, los cuales son asumidos como vulnerabilidades de la empresa que pueden afectar la satisfacción de los clientes. Tales debilidades son las llamadas brechas del servicio y coexisten dentro de la empresa. De ellas hemos identificado cinco:

Brecha uno. Expectativa de los clientes versus percepción de la alta gerencia: Existen diferencias entre lo pensado -léase expectativas de clientes - y la realidad; pero además esta brecha subsiste cuando la gerencia no anticipa el deseo de los clientes o los valores agregados esperados en el servicio deseado.

Brecha dos. Apreciación por parte de la administración o gerencia versus desempeños reales del personal en materia de calidad del servicio brindado: Hay una incapacidad por la gerencia al establecer especificaciones de calidad acorde a requisitos. Ello existe a partir de factores como restricción de recursos, cambios o condiciones no evaluadas del mercado.
Brecha tres. Complementos, detalles, especificaciones asociadas a la calidad planteada versus realidad en las estrategias del servicio: Este contraste sucede entre las especificaciones de calidad, visibilizadas en las funciones de cada cargo y la diversidad de grado de calidad del servicio, el cual no es brindado adecuadamente. Esto se da a partir del rendimiento de cada colaborador o empleado, el cual influye de manera decisoria en el servicio, cuyo comportamiento individual no puede ser normalizado.

Brecha cuatro. Realización de los diferentes tipos de servicio versus eficacia en la comunicación externa: Ello ocurre cuando la comunicación externa afecta o influencia las expectativas del cliente, en otras palabras, cuando la empresa ofrece más o excede sus expectativas en relación de lo que puede ofertar.

Brecha cinco. Servicio esperado versus servicio percibido por el cliente: Implica el esfuerzo para asegurar calidad en el servicio, y que ha de alcanzar en materia de satisfacción, o exceder la confianza, seguridad y expectativa en cada uno de los clientes.

Por su parte, Zeithaml, Berry y Parasuraman (1988) asumieron otros criterios, de los cuales se desprende que el peso de las citadas brechas en la calidad del servicio es mayor en la brecha cinco, la cual se identifica como función de las cuatro brechas explicitadas. Es el cliente quien, sobre la base cultural de experiencias vividas y la curiosidad de nuevas emociones, recrea sus expectativas personales, las cuales se fundamentan en experiencias vividas, perspectivas de terceros y criterios verbales. Reforzando esta idea, Parasuraman, Berry y Zeithaml (1993) enfatizan en que si las expectativas exceden las percepciones, entonces el servicio no resulta de calidad; si las percepciones igualan a las expectativas, el servicio es satisfactorio; y si el servicio excede a las percepciones, el campo es el ideal.

De manera análoga, Cantú (2006) argumenta que la satisfacción del cliente se debe asumir como la "percepción de los clientes externos relativo a los productos y servicios brindados por la empresa" ( $p$. 15). Estos clientes persiguen las evidencias sobre las medidas o índices claves en la empresa a la hora de evaluar el desempeño, siempre orientado a la mejora continua y estado de excelencia. A partir de la visión de un sistema para gestionar la calidad, Colín (2002) indica que la satisfacción del cliente se encuentra implícita, pues es tomada como "la percepción del mismo sobre el grado en que se han cumplido sus requisitos" (p. 4). 
Aquellas entidades orientadas a la satisfacción de los clientes definen con éxito la calidad de forma operativa, son varias las técnicas orientadas a determinarla, las cuales pueden ser ejecutadas de manera sutil o evidente. A través de ellas se impone el compromiso de tiempo en la gestión de la servucción, mientras otras focalizan su empeño en la supervisión y monitoreo de las necesidades y actitudes de los clientes, así como bien lo reflexiona Denton (1991). Retomando a Parasuraman, Berry y Zeithaml (1993), estos autores sugieren cinco dimensiones implícitas en el modelo SERVQUAL: la tangibilidad, expresada como la apariencia y estado de las instalaciones físicas, el profesionalismo de los colaboradores en la empresa o personal y el grado de efectividad comunicacional y relacional; la fiabilidad, asumida como la competencia y capacidad demostrada para entregar el servicio; la capacidad de respuesta, visibilizada como la voluntad de arrimar el hombro a los clientes, brindando servicio rápido; la seguridad, expresada en la asertividad, el conocimiento, la gentileza y la cortesía de los colaboradores de la empresa, capacidad individual de transmitir cordialidad y seguridad; y, por último, la empatía, cuestión aún incomprendida y que resulta de la relación interpersonal, la proyección de cuidado y el enfoque individualizado a cada cliente.

No obstante, frente a lo señalado, los autores Cronin y Taylor (1992) debaten los atributos en la medición de las expectativas de la calidad del servicio. Estos investigadores basan su estudio en la tendencia de los clientes a través de los niveles de expectativas, cuyos resultados en los niveles de percepción raramente superan a estas. Asimismo, ellos definen la calidad de servicio como "función exclusiva de la percepción del servicio" (p. 11), de esta forma recrean el instrumento denominado SERVPERF (service performance), el cual está estrictamente basado en las percepciones. Este modelo toma veintidós afirmaciones referidas a percepciones del desempeño recibido según el modelo SERVQUAL. El modelo SERVPERF destaca en la manera en que reduce en la mitad a las mediciones versus SERVQUAL y es calculado en función de la suma de las percepciones ya explicitadas. Además, permite visibilizar la relación proporcional entre la calidad del servicio y la suma de dichas percepciones. Como complemento, el modelo SERVQUAL es valorado, según Parasuraman, Berry y Zeithaml (1993), por permitir la medición de la calidad de un servicio a partir de la diferencia entre percepciones y expectativas de los clientes frente a las dimensiones del servicio. La presente investigación pretende combi- nar ambos enfoques a la hora de complementar el concepto más exacto de calidad en los servicios.

De este modo, se reconoce la solución práctica del modelo SERVQUAL y se identifican ventajas y variables, además de la versatilidad al ponderar rasgos subjetivos implícitos en la calidad y el servicio. Es de señalar también que las desventajas provocan la reflexión a la hora de la obtención de resultados más cercanos a la realidad asociados a personas y colectivos en un contexto propio laboral o personal, en el que viajan y se hospedan, donde se determinan las variables asumidas como relevantes en la calidad del servicio.

Este estudio concluye con la necesidad de aplicar el modelo SERVQUAL, el cual atiende al criterio de la estacionalidad turística, al destino y al perfil del turista, todo ello orientado a elevar la confiabilidad de los resultados. Además, se menciona que no existen los llamados "clientes cautivos" ni el resultado multivariante y complejo de calcular, así como tampoco existe el grado de la fidelidad de los clientes. Entre otros factores, se citan la variedad de destinos existentes, la cantidad y la variedad de servicios de los destinos, lo cual contribuye al abanico de expectativas que varía en cada persona. La hipótesis esgrimida en esta investigación se centra en comprobar la validez de un constructo para determinar la calidad de los servicios turísticos y, también, para que exista un criterio más objetivo y adecuado de la calidad integral percibida de los servicios.

\section{METODOLOGÍA}

El tipo de investigación es descriptivo porque realiza un diagnóstico de la variable: la calidad de los servicios turísticos. Su enfoque es cuantitativo y cualitativo porque precisa atender los objetivos planteados en el estudio. Este trabajo es propuesto bajo el criterio de las visiones de expectativas y percepciones en la calidad de servicios turísticos, el cual surge al calcular la muestra representativa de una población de 371 turistas con los servicios turísticos ya recibidos. Desde la visión de la estadística inferencial, se obtiene la herramienta de la encuesta apelando a la escala de Likert, donde se adecúan aseveraciones y preguntas a las dimensiones y variables implícitas en la calidad turística. Luego de realizado el estudio de fiabilidad y validez del cuestionario previo a su aplicación, se solicita el uso de los métodos inductivo, deductivo, analítico, sintético y sistémico. El diseño de investigación es no experimental y, sobre todo, correlacional de corte longitudinal, debido a que se ejecuta más de una 
medición. Se considera una investigación de tipo no experimental por no inferir ni manipular el estudio, además se pretende descubrir las relaciones de la calidad en cada dimensión y su interrelación con los servicios turísticos. La unidad de análisis va en relación con turistas que han hecho uso de los servicios ofrecidos en Riobamba, provincia de Chimborazo, Ecuador.

\section{Codificación del instrumento de medición}

Se muestra a continuación la codificación del instrumento: la calidad de los servicios turísticos correspondiente a expectativas y percepciones. Asimismo, se ha depurado y validado para poder ser aplicado en encuestas a 371 turistas que llegaron a Riobamba. Cada ítem tiene un código relacionado con el instrumento al que corresponde, así como también la dimensión que la aborda. En la Tabla 1 se puede apreciar las dimensiones de la variable "Calidad de los servicios turísticos" en base al modelo SERVQUAL original, ajustado a la realidad de Riobamba, donde además se identifica la codificación de cada uno de los ítems considerados por cada una de las cinco dimensiones.
En esta investigación se parte del enfoque en el que todo procesamiento estadístico inferencial, desde el abordaje del análisis de fiabilidad y consistencia hasta la escala de aptitud tipo Likert, provee un sesgo de confiabilidad al aplicarse al cuestionario. Tal presunción surge del estadígrafo denominado alfa de Cronbach, lo cual estima la fiabilidad del instrumento estadístico, analizando el conjunto de ítems considerados a medir las dimensiones implícitas en el constructo teórico.

A partir del uso del estadígrafo alfa de Cronbach se obtiene que los ítems previos ya medidos a través de la escala de Likert, capaces de medir el constructo, demuestran su grado de correlación. Ahora, si asumimos los presupuestos de Frías (2014), a mayor cercanía del valor del alfa al valor 1 , se muestra la estabilidad interna de cada uno de los ítems ensayados. Esta fiabilidad ha de calcularse a partir de los datos implícitos de cada muestra tomada, garantizando el valor fiable implícito en la medida del constructo, a partir de la muestra calculada en la investigación. Son sugeridas las recomendaciones al evaluar los coeficientes obtenidos

Tabla 1. Codificación del instrumento relativo al análisis de la calidad implícita en los servicios turísticos de Riobamba.

\begin{tabular}{|c|c|c|}
\hline Dimensión & Código & Calidad de los servicios turísticos \\
\hline \multirow{4}{*}{ Elementos tangibles } & ET1 & Tecnología moderna \\
\hline & ET2 & Atracción visual en cada instalación \\
\hline & ET3 & Aspecto del personal \\
\hline & ET4 & Elementos tangibles atractivos: folletería \\
\hline \multirow{5}{*}{ Fiabilidad } & FB1 & Observancia de promesas \\
\hline & FB2 & Compromiso y equidad al resolver problemas \\
\hline & FB3 & Desempeño en el servicio, que debe ser de primera \\
\hline & FB4 & Consumación en el término acordado \\
\hline & FB5 & Inexistencia de errores \\
\hline \multirow{4}{*}{ Capacidad de respuesta } & CR1 & Personal comunicativo \\
\hline & CR2 & Rapidez del personal \\
\hline & CR3 & Personal colaborador \\
\hline & CR4 & Personal informado \\
\hline \multirow{4}{*}{ Seguridad } & SG1 & Personal transmite confianza \\
\hline & SG2 & Turistas seguros con su proveedor \\
\hline & SG3 & Personal amable \\
\hline & SG4 & Personal bien formado \\
\hline \multirow{5}{*}{ Empatía } & EM1 & Solicitud personalizada al turista \\
\hline & EM2 & Itinerario ventajoso \\
\hline & EM3 & Servicio individualizado a los copartícipes \\
\hline & EM4 & Inquietud ante solicitudes del clientes \\
\hline & EM5 & Intuición y alta comprensión de necesidades del turista \\
\hline
\end{tabular}

Fuente: Elaboración propia. 
del estadígrafo de alfa de Cronbach —en adelante llamado $\mathrm{AC}$ - . Así, queda señalar que: $\mathrm{AC}>9$ es "excelente"; $A C>8$, "bueno"; $A C>7$, "aceptable"; $A C>6$, "cuestionable; $A C>5$, "pobre"; y $A C<5$, "inaceptable".

Los resultados al evaluar la calidad son expuestos, configurando las dimensiones implícitas para medir cada constructo de las variables analizadas. Estas cinco dimensiones son necesarias a la hora del constructo general de la calidad de los servicios turísticos. De esta forma, se extrae el alfa de Cronbach, compuesto por los 44 ítems que corresponden a expectativas (22 ítems) y percepciones (22 ítems). Además, analiza la variable calidad del servicio y cada una de sus dimensiones, en función de las expectativas y percepciones de los clientes.

En la Tabla 2, es apreciable el valor de "excelente" asociado al constructo de la calidad de los servicios turísticos de la ciudad de Riobamba.

Tabla 2. Coeficiente $A C$ del constructo calidad de los servicios turísticos de Riobamba.

\begin{tabular}{|l|c|c|}
\hline Constructos & AC & $\mathbf{N} .^{\circ}$ de elementos \\
\hline Expectativas & 9,64 & 22 \\
\hline Percepciones & 9,42 & 22 \\
\hline Expectativas y percepciones & 9,35 & 44 \\
\hline
\end{tabular}

Fuente: Elaboración propia.

En la Tabla 3 se evidencia el AC asociado al constructo calidad de los servicios turísticos de Riobamba obtenido mediante el paquete SPSS (versión 23) y correspondiente a las expectativas con un valor excelente.

Tabla 3. AC de la calidad de los servicios turísticos de Riobamba: expectativas.

\begin{tabular}{|c|c|}
\hline $\mathbf{A C}$ & $\mathbf{N} .^{\circ}$ de elementos \\
\hline 9,64 & 22 \\
\hline
\end{tabular}

Fuente: Elaboración propia.

En la Tabla 4 se evidencia el AC asociado del constructo calidad de los servicios turísticos de Riobamba obtenido mediante el paquete SPSS (versión 23) y correspondiente a las percepciones con un valor excelente.

Tabla 4. AC de calidad de los servicios turísticos: percepciones

\begin{tabular}{|c|c|}
\hline AC & $\mathbf{N}^{\circ}$ de elementos \\
\hline 9,42 & 22 \\
\hline
\end{tabular}

Fuente: Elaboración propia.
Se exponen los resultados de AC de las dimensiones correspondientes a la variable de percepciones del constructo: calidad de los servicios turísticos de Riobamba específicamente. Así pues, en la Tabla 5 se evidencian los valores obtenidos mediante el paquete SPSS (versión 23), los cuales son aceptables y buenos.

Tabla 5. AC de calidad de los servicios turísticos: percepciones por dimensiones.

\begin{tabular}{|l|c|c|}
\hline Dimensión & AC & $\mathbf{N} .^{\circ}$ de elementos \\
\hline Elementos tangibles & 7,66 & 4 \\
\hline Fiabilidad & 8,22 & 5 \\
\hline Capacidad de respuesta & 8,12 & 4 \\
\hline Seguridad & 7,86 & 4 \\
\hline Empatía & 8,72 & 5 \\
\hline
\end{tabular}

Fuente: Elaboración propia.

El valor obtenido de AC en la variable calidad de los servicios turísticos es $>0,7$, es decir, resulta excelente. Ello determina la confiabilidad del instrumento aplicado a tenor de la teoría estadística, analizando además cada ítem de la variable independiente con la dimensión. Asimismo, estos son, como la media y la varianza, calculados mediante estadígrafos de dispersión de tendencia central. Luego, se calcula la correlación total de elementos corregidos y el AC de haberse suprimido de los ítems del constructo calidad de los servicios. También se aplica con el propósito de buscar factores o dimensiones subyacentes en el análisis $\mathrm{y}$, a partir del modelo teórico o genérico, un análisis factorial exploratorio. Relativo a la matriz de correlaciones, se infiere que a mayor valor, las correlaciones dentro de la matriz son bajas; mientras que a menor valor del determinante, resultan correlaciones altas en la matriz.

Así es el caso resultante en la investigación y ello evidencia un determinante ( $D=9,367 E-6)$. Por su parte, la varianza total explicada es ofertada con el conjunto de los autovalores de la matriz correspondiente a las varianzas y las covarianzas, relacionada con la proporción porcentual de la varianza calculada representada en cada uno. De tal manera, formulan la varianza total expresada por cada componente, además de los porcentajes de varianza asociados a cada factor. Estos se obtienen al dividir el autovalor entre la sumatoria de estos, por lo que se enfatiza en la necesaria coincidencia con el total de variables.

Son extraídos por defecto factores como autovalores $>1$, el cual tiene la matriz analizada. En este caso, como se parte de un modelo hipotético, se aplicó un análisis factorial confirmatorio (AFC), por 
lo que limitamos el modelo a que solo determine tres factores o dimensiones. Estos explican el $62,8 \%$ de la varianza para el caso de las expectativas y $55,7 \%$ de las percepciones, cuya calidad del servicio es calculado a partir de la relación expectativas versus percepciones por la varianza total explicada (VTE), todo lo cual se evidencia en la Tabla 7. Partiendo de este punto, sería factible determinar si son esas las verdaderas dimensiones o factores.

La Tabla 6 muestra la media y desviación estándar de cada uno de los elementos de la variable independiente "Calidad de los servicios turísticos", lo cual indica cuánto pueden alejarse los valores respecto al promedio o media.

En la Tabla 8, la denominada prueba de Bartlett es reflejada. Allí se correlacionan las expectativas y percepciones, obteniéndose valores de 0,955 y 0,848 , considerados valores excelentes, y un nivel de significación de 0,000. De tal manera, se rechaza la hipótesis de no-correlación de las variables, así se justifica la adecuación del análisis factorial, por cuanto el resultado de Kaiser-Meyer-Olkin (KMO) es $>0,5$. Este valor es asumido como umbral generalmente aceptado (UGA).

\section{RECOMENDACIONES}

- Incentivar el análisis y la aplicación de estrategias que ayuden a relacionar expectativas y percepciones en la calidad de los servicios turísticos, como hemos realizado con el destino turístico Riobamba, con soporte y sustentación estadística.

- Considerar para próximas investigaciones las dimensiones de las variables analizadas en este estudio y tomar en cuenta el aporte significativo que brindan las dimensiones de la calidad de los servicios turísticos.

- Involucrar el grado de importancia que existe entre las expectativas y percepciones de la calidad de los servicios turísticos en la construcción de modelos estadísticos para la medición de calidad en destinos turísticos,

Tabla 6. Calidad de los servicios turísticos: media y desviación estándar de todos los elementos.

\begin{tabular}{|c|c|c|c|}
\hline \multicolumn{4}{|c|}{ Estadísticos descriptivos } \\
\hline Calidad de los servicios turísticos & Media & $\begin{array}{c}\text { Desviación } \\
\text { estándar }\end{array}$ & N. ${ }^{\circ}$ de análisis \\
\hline ET1: Tecnología moderna & 3,57 & 0,807 & 371 \\
\hline ET2: Atracción en las instalaciones & 3,67 & 0,865 & 371 \\
\hline ET3: Apariencia del personal & 3,69 & 0,900 & 371 \\
\hline ET4: Folletería atractiva & 3,50 & 0,954 & 371 \\
\hline FB1: Cumplimiento de lo prometido a tiempo & 3,57 & 0,908 & 371 \\
\hline FB2: Interés por solucionar el problema & 3,54 & 0,973 & 371 \\
\hline FB3: Cumplimiento del servicio a la primera & 3,45 & 1,026 & 371 \\
\hline FB4: Prestación del servicio a tiempo & 3,57 & 0,962 & 371 \\
\hline FB5: Registro exento de errores & 3,66 & 0,935 & 371 \\
\hline CR1: Personal informa cuando finaliza el servicio & 3,59 & 0,941 & 371 \\
\hline CR2: Personal ofrece servicio rápido & 3,64 & 0,885 & 371 \\
\hline CR3: Personal siempre dispuesto & 3,60 & 0,943 & 371 \\
\hline CR4: Personal nunca ocupado para responder preguntas & 3,71 & 0,967 & 371 \\
\hline SG1: Personal transmite confianza a clientes & 3,67 & 0,916 & 371 \\
\hline SG2: Confianza en negociación con organización & 3,64 & 0,977 & 371 \\
\hline SG3: Personal amable con los turistas & 3,61 & 0,965 & 371 \\
\hline SG4: Personal con suficiente conocimiento para servir & 3,64 & 0,946 & 371 \\
\hline EM1: Empresa con atención individualizada & 3,56 & 0,944 & 371 \\
\hline EM2: Horario de atención orientado al turista & 3,52 & 0,976 & 371 \\
\hline EM3: Atención personalizada del personal & 3,53 & 0,953 & 371 \\
\hline EM4: Empresa se preocupa por intereses del turista & 3,56 & 0,972 & 371 \\
\hline EM5: Personal comprende necesidades específicas & 3,53 & 1,043 & 371 \\
\hline
\end{tabular}

Fuente: Elaboración propia. 
Tabla 7. Calidad del servicio calculado a partir de la relación expectativas versus percepciones a por la varianza total explicada (VTE).

\begin{tabular}{|c|c|c|c|c|c|c|}
\hline \multirow{2}{*}{ Expectativas } & \multicolumn{3}{|c|}{ Autovalores calculados iniciales } & \multicolumn{3}{|c|}{ Sumas de extracción de varianzas (V) } \\
\hline & Total & $\%$ varianza & $\%$ acumulado & Total & $\%$ varianza & $\%$ acumulado \\
\hline 1 & 12,556 & 57,072 & 57,072 & 12,556 & 57,072 & 57,072 \\
\hline 2 & 1,258 & 5,716 & 62,788 & 1,258 & 5,716 & 62,788 \\
\hline 22 & 0,149 & 0,679 & 100,000 & & & \\
\hline \multirow{2}{*}{ Percepciones } & \multicolumn{3}{|c|}{ Autovalores } & \multicolumn{3}{|c|}{ Sumatorias de rotación de las cargas al cuadrado } \\
\hline & Total & $\%$ varianza & $\%$ acumulado & Total & $\%$ varianza & $\%$ acumulado \\
\hline 1 & 9,935 & 45,159 & 45,159 & 4,570 & 20,773 & 20,773 \\
\hline 2 & 1,221 & 5,550 & 50,709 & 3,876 & 17,617 & 38,390 \\
\hline 3 & 1,087 & 4,943 & 55,652 & 3,798 & 17,263 & 55,652 \\
\hline 22 & 0,252 & 1,147 & 100,000 & & & \\
\hline
\end{tabular}

Fuente: Elaboración propia.

Tabla 8. Constructo calidad de los servicios turísticos: KMO y Bartlett.

\begin{tabular}{|c|c|c|}
\hline \multicolumn{2}{|c|}{ Prueba de KMO y Bartlett: Expectativas } \\
\hline \multirow{2}{*}{ Medida KMO de adecuación de muestreo } & 0,955 \\
\hline \multirow{2}{*}{ Prueba de esfericidad de Bartlett } & Aproximación chi-cuadrado & 6369,491 \\
\cline { 2 - 3 } & Grados de libertad & 231 \\
\cline { 2 - 3 } & Significación & 0,000 \\
\hline \multicolumn{2}{|c|}{ Prueba de KMO y Bartlett: Percepciones } \\
\hline Medida KMO de adecuación de muestreo & 0,948 \\
\hline \multirow{2}{*}{ Prueba de esfericidad de Bartlett } & Aproximación chi-cuadrado & 4189,418 \\
\cline { 2 - 3 } & Grados de libertad & 231 \\
\cline { 2 - 3 }
\end{tabular}

Fuente: Elaboración propia.

puesto que ambos son referentes de la relación oferta-demanda que sustenta la satisfacción de los turistas y el desarrollo local.

\section{CONCLUSIONES}

- Se han calculado la validez y la fiabilidad del instrumento, ratificando excelentes resultados mediante el KMO (valores altos cercanos a uno).

- Tal resultado garantiza la adecuación del análisis factorial al validar ambos instrumentos de medición.

- La fiabilidad de ambos instrumentos es calculada mediante el alfa de Cronbach (AC) por dimensión, tributando excelentes resultados. En todos los casos, este coeficiente resulta $>0,7$, lo cual garantiza que son instrumentos de medición estables.
- A partir de las respuestas al aplicar los instrumentos, es destacable que los turistas determinen expectativas mayores a las percepciones; aunque se traten de respuestas bajo cierto criterio, ello permite ejecutar el análisis de la satisfacción.

- La investigación permite inferir la relación entre expectativas y percepciones de la calidad de los servicios turísticos, sustentado con la prueba del KMO.

- Los valores determinados en el alfa de Cronbach (AC) fueron calculados apelando al análisis de tipo factorial, el cual es adecuado a la hora de validar los instrumentos.

- Se demuestra que las dimensiones aportan en favor de sus constructos, desde donde se destaca la fiabilidad para la calidad de los servicios turísticos. 


\section{REFERENCIAS BIBLIOGRÁFICAS}

[1] Cantú, H. (2006). Desarrollo de una cultura de calidad (3 ed.). México D. F., México: McGraw-Hill.

[2] Castelluci, D. (2009). La calidad y los servicios en destinos turísticos maduros. Aportes y Transferencias, 13(2), 29-44. Recuperado de http://www.redalyc.org/pdf/276/27621943003.pdf

[3] Colín, L. (2002). Las normas ISO 9000:2000 de Sistemas de Gestión de la Calidad. Boletín IIE, 26(4), 182-188. Recuperado de https://www. ineel.mx/bollsO02/tecni2.pdf

[4] Cronin, J. y Taylor, S. (1992). Measuring Service Quality: A Reexamination and Extension. Journal of Marketing, 56(3), 55-68.

[5] Denton, K. (1991). Calidad en el servicio a los clientes. Texas, Estados Unidos: Díaz de Santos.

[6] Frías, D. (2014). Apuntes de SPSS: consistencia interna. Recuperado de https://es.scribd.com/ document/355502616/ApuntesSPSS-friasnavas-pdf

[7] Olmedo, K. (2014). Evaluación de la calidad del servicio turístico de los guías de los museos del Centro Histórico de Quito (CHQ) $y$ propuesta de mejoras. (Tesis de pregrado). Pontificia Universidad Católica del Ecuador, Quito. Recuperado de http://repositorio.puce. edu.ec/handle/22000/9929

[8] Parasuraman, A., Berry, L. y Zeithaml, V. (1988). SERVQUAL: A Multiple-Item Scale for
Measuring Consumer Perceptions of Service Quality. Journal of Retailing, 64(1), 12-40.

[9] Parasuraman, A., Berry, L. y Zeithaml, V. (1993). More on improving service quality measurement. Journal of Retailing, 69(1), 140147.

[10] Peñaloza, L., Toulouse, N. y Visconti, L. (eds.) (2007). Marketing Management: A Cultural Perspective. Londres, Reino Unido: Rotledge.

[11] Pride, W. y Ferrell, O. (1997). Marketing. Conceptos y estrategias. México D. F., México: McGraw-Hill.

[12] Romani, M. (2017). Calidad turística y buenas prácticas de servicios turísticos en Lima Metropolitana, 2016. (Tesis de maestría). Universidad César Vallejo, Lima. Recuperado de http://repositorio.ucv.edu.pe/ bitstream/handle/UCV/9084/Romani_NML. pdf? sequence $=1$ \&isAllowed $=y$

[13] Stanton, W., Etzel, M. y Walker, B. (2004). Fundamentos de marketing. México D. F., México: McGraw-Hill. Recuperado de https:// mercadeo1marthasandino.files.wordpress. com/2015/02/fundamentos-de-marketingstanton-14edi.pdf

[14] Zeithaml, V., Berry, L. y Parasuraman, A. (1988). Communication and Control Processes in the Delivery of Service Quality. Journal of Marketing, 52(2), 35-48.

[15] Zeithaml, V., Bitner, M. y Gremler, D. (2005). Services marketing. Integrating Customer Focus Across the Firm (4 ed.). México D. F., México: McGraw-Hill. 


\section{Validation of a construct to determine the quality of tourism services}

Diego Mauricio Calvopiña Andrade ${ }^{1}$

\begin{abstract}
This study describes the validation of a construct to determine the quality of tourism services, which is regarded as the activity with the greatest global impact on political, economic, social, cultural, environmental and technological fields. This activity is no longer only based on the idea of the trip as it was previously understood. At present, it is increasingly oriented towards an active and participatory model, which suggests establishing innovative coexistence, learning and enjoyment strategies for both travelers and hosts. To this end, it is essential to consider services and their impact on competitiveness and sustainable development. The current trend of tourism is to place greater emphasis on the quality of tourism services as it is considered to be an essential part of the tourist experience, which raises the following question: what dimensions from the quality of tourism services construct (perceptions) influence tourism services? The validity and reliability of the instrument was determined, thus upholding its adequacy and the impact on tourism service quality criteria.
\end{abstract}

Keywords: Construct; SERVQUAL; quality; tourism services.

\section{INTRODUCTION}

This study is based on tourism service quality theories and models, such as the National Plan of Good Living of Ecuador, the strategic tourism plan PLANDETUR 2020 and the tourism plans of Chimborazo province and the city of Riobamba, in particular. The conformance of this study with the objectives stated in the Millennium Development Goals, the National Objectives for Good Living, the Código Orgánico de Ordenamiento Territorial, Autonomía y Descentralización ${ }^{\mathrm{TN} 1}$ (COOTAD) and the global and Ecuadorian tourism regulations is evident, as a social contribution. For Pride and Ferrell (1997) "es importante destacar que la calidad es determinante en la percepción del cliente sobre un servicio" [it is important to emphasize that quality is a decisive factor in the customer's perception of a service] (p. 35); these authors also define service quality "como la percepción que tienen los clientes si un servicio satisface o si excede sus expectativas" [as the perception that customers have if a service satisfies or exceeds their expectations] (p. 12). Based on the above, two key attributes within service quality perception have been characterized. Also, the precepts of Stanton, Etzel and Walker (2004), Castelluci (2009) and Romani (2017) have been adopted. The first precept considers that service quality is determined by the customer (not the service experience agent or service provider); whereas the second results from comparing and contrasting initial customer expectations, who categorize and evaluate the quality of the service received, against real perceptions. These expectations are based on mental models acquired from previous experiences or prevailing advertising.

Meanwhile, the researchers Parasuraman, Berry and Zeithaml (1988) consider other precepts, stating that customers' expectations are the decisive factor in the quality evaluation of a service offered by a specific establishment. This happens because customers measure, compare and evaluate the offered service according to their expectations. The overall perception of the

\footnotetext{
1 Master in Industrial Engineering from the Universidad Nacional Mayor de San Marcos. Professor at the Universidad Nacional de Chimborazo. Chimborazo, Ecuador. E-mail: dcalvopina@unach.edu.ec

ORCID: 000-0003-4034-4185.

Google Scholar: https://scholar.google.es/citations?view_op=new articles\&hl=es\&imq=Diego + Mauricio + Calvopi $\% \mathrm{C} 3 \% \mathrm{~B} 1 \mathrm{a}+$ Andrade.

TN1: Legislation that establishes the political-administrative organization of Ecuadorian territory.
} 
received service then results from the contrast between expected service versus perceived service.

Regarding the service quality approach, the authors Zeithaml, Bitner and Gremler (2005) indicate that "la calidad de servicio es un componente primordial de las percepciones del cliente" [service quality is an important component of customers' perceptions] ( $p$. 7). On the other hand, scholars Olmedo (2014) and Romani (2017) state that it the question which prevails in the customers' mind, as "la calidad del servicio también puede ser fundamental para determinar la satisfacción del cliente" [service quality can also be essential to determine customer satisfaction] ( $p$. 65). Additionally, Peñaloza (2007) clearly states that quality is relevant because "el fin último del marketing es la satisfacción del cliente y esta es posible solo cuando se proporcionan productos y servicios de calidad, los cuales están íntimamente relacionados, motivo por el que resulta obligatorio centrarse en la valoración de la calidad" (p. 36) [customer satisfaction is the ultimate goal of marketing, and it is only achieved when quality goods and services, which are closely related, are provided, which is why it is therefore mandatory to focus on quality evaluation].

Similarly, Parasuraman, Berry and Zeithaml (1988) presented the SERVQUAL (service quality) model, a research instrument that works by establishing the degree of customer satisfaction (of a service-oriented company) through the existing gaps between expected and received service. The concept suggested by the authors is complex, as the assessment and questioning of service quality is subjective. Thus, the approach is different for tangibles and service-production, where customers have physical elements to differentiate and judge the quality level of service received.

The aforementioned researchers address several critical issues, which are seen as vulnerabilities in companies that may affect customer satisfaction. Said weaknesses are known as service gaps that coexist within the company. Of these, we have identified five:

Gap one. Customer expectations vs senior management perception: There are differences between the presumed-read: "customer expectations"-and reality; this gap also persists when management fails to anticipate customer desires or the aggregate values expected to be found in the desired service.

Gap two. Management appraisal vs actual personnel performance regarding offered service quality: Management fails to establish quality specifications according to requirements. This arises from factors such as limited resources, changes in the market or non-evaluated market conditions.

Gap three. Complements, details and specifications related to proposed quality vs reality in service strategies: This contrast occurs between quality specifications, perceivable in the functions of each position, and the variety in the degree of service quality. This is based on the performance of each collaborator or employee, whose behavior cannot be standardized, which has a considerable impact on service.

Gap four: Different services delivery vs effective external communication: This occurs when external communication affects or influences customer expectations, that is, when the company offers more than it can deliver.

Gap five. Expected service vs perceived service: This involves the effort made to ensure service quality and to exceed customer trust, safety and expectations in terms of customer satisfaction.

Zeithaml, Berry and Parasuraman (1988) considered other criteria, from which it is understood that gap five is the most important gap regarding service quality, as it is identified on the basis of the other four. It is the customers who form their personal expectations on the basis of personal and cultural experiences, as well as the perspectives and opinions of others. To support this idea, Zeithaml, Berry and Parasuraman (1993) stress that if expectations exceed perceptions, then service is deemed as not quality service; if perceptions match expectations, the service is satisfactory; and if the service exceeds perceptions, then it is ideal.

Similarly, Cantú (2006) states that customer satisfaction should be understood as the "percepción de los clientes externos relativo a los productos $y$ servicios brindados por la empresa" [perceptions of external customers regarding goods and services provided by a company] (p. 15). These customers look for information about ratings and rankings when evaluating Company performance, always in regard to continuous improvement and excellence. Based on the idea of a quality management system, Colín (2002) points out that customer satisfaction is implicit, as it is considered as "its perception about the extent to which they have met their requirements" (p. 4).

Customer-oriented companies successfully define quality in an operative manner, using different tech- 
niques that can be applied in a subtle or evident manner to determine customer satisfaction. Some techniques require a commitment regarding service-production management; whereas others are focused on the supervision and monitoring of the customers' needs and attitudes, as explained by Denton (1991). Authors Parasuraman, Berry and Zeithmal (1993) suggest five dimensions implicit in the SERVQUAL model: tangibles, understood as the appearance and state of the physical facilities, professionalism of the personnel and collaborators and the degree of effective communications and relations; reliability, understood as the ability to perform the service; responsiveness, understood as the willingness to assist customers by providing services promptly; assurance, expressed through the assertiveness, knowledge, curtsey and politeness of employees and their capacity to convey hospitality and confidence; and lastly, empathy, a subject yet to be understood that results from interpersonal relationships, the provision of caring and individualized attention to customers.

Nevertheless, Cronin and Taylor (1992) discuss the attributes considered for the measurement of service quality expectations. These researchers base their study on customer tendencies by means of expectation levels, whose perception results rarely surpass expectations. Likewise, said authors define quality as "función exclusiva de la percepción del servicio" [an exclusive function of service perception] (p. 11) and designed the instrument called SERVPERF (service performance), which is strictly based on perceptions. It takes 22 statements regarding performance perception from the SERVQUAL model. The SERVPERF is notable because it reduces by half the number of items to be measured and it performs service quality measurement, and is calculated on the basis of the sum of the perceptions. In addition, it makes it possible to visualize the proportional relationship between service quality and the sum of perceptions. As a complement, the SERVQUAL model is valued, according to Parasuraman, Berry and Zeithaml (1993) for enabling the measurement of service quality based on the difference between customers' perceptions and expectations regarding service dimensions. This study attempts to combine both approaches in order to achieve a more precise concept of service quality.

Thus, the practicality of SERVQUAL model is recognized, advantages and variables are identified, and its versatility to weigh subjective features implicit in quality and service is also recognized. It should be noted that the disadvantages are considered when the aim is obtaining more reliable results, associated to individuals and collectives in working or personal environments, in which they travel and stay at lodgings, where the relevant variables in relation to quality service are determined.

This research study concludes that it is necessary to apply the SERVQUAL model, which addresses tourism seasonality, destination and tourist profile criterion, aimed at raising results reliability. Additionally, it is mentioned that there are neither "captive customers" nor degree of customer loyalty. The number and variety of destinations, among other factors, contribute to the range of expectations of each person. The hypothesis of this study is focused on validating a construct that serves to determine tourism service quality and, also, to generate a more objective and adequate criterion to measure perceived service quality in a comprehensive manner.

\section{METHODOLOGY}

This is a descriptive investigation because it examines the variable "tourism service quality". Its focus is quantitative and qualitative because it attempts to fulfill the objectives set forth in the study. This study considers expectations and perceptions in service quality as evaluation criteria, which arise when calculating the representative sample of 371 tourists who had already received tourist services. The instrument used, a survey questionnaire where questions and statements are adapted to the dimensions and variables implied in tourism quality, was designed using the Likert scale. After conducting the reliability and validity analysis of the questionnaire prior to its application, inductive, deductive, analytical, synthetic and systemic methods were used. A non-experimental, cross-sectional and correlational design was used for this research, as more than one measurement is performed. It is non-experimental because there was no manipulation or interference, moreover, it aims to discover the relationships of quality in each dimension and its interrelation with tourism services. The analysis unit is comprised of tourists that have made use of the services offered in Riobamba, Chimborazo Province, Ecuador.

\section{Measuring instrument design}

Shown below is the coding of the instrument: tourism services quality regarding expectations and perceptions. It was refined and validated in order to be applied to surveys given to 371 tourists that visited Riobamba. Each item has a code related to the instrument to which it corresponds, as well as to the dimension to which it belongs. All dimensions of variable "Tourism services quality" are displayed in 
Table 1, based on the original SERVQUAL model, adjusted to Riobamba's conditions and identified by codes determined for each one of the five variables.

In this investigation, it is considered that the use of inferential statistical processing, from reliability and consistency analysis to Likert scale, creates a reliability bias in the questionnaire. This presumption arises from the statistic Cronbach's alpha, which estimates the reliability of the instrument analyzing the set of items to measure the dimensions implicit in the theoretical construct.

Cronbach's alpha (CA) makes it possible to establish the degree of correlation between the items, capable of measuring the construct, measured by means of the Likert scale. According to Frías (2014), the greater proximity of alpha value to 1 shows the internal stability of the tested items. This reliability is to be calculated from implicit data of each sample, thus guaranteeing the reliable value of the construct based on the research sample. The coefficient values are: CA>9, "excellent"; $C A>8$, "good"; $C A>7$, "acceptable"; CA>6, "questionable"; $C A>5$, "weak"; and $\mathrm{CA}<5$, "inacceptable".
The results obtained from the evaluation of quality are presented, configuring the implicit dimensions to measure each construct. These five dimensions are necessary when creating the general construct "tourism services quality". Thus, Cronbach's alpha is obtained from the 44 items corresponding to expectations (22 items) and perceptions (22 items). In addition, tourism services quality variable and each of its dimensions are analyzed on the basis of customers' expectations and perceptions.

In Table 2, the "excellent" value is associated with the construct tourism services quality in Riobamba.

Table 2. Cronbach's alpha of the construct tourism services quality in Riobamba.

\begin{tabular}{|l|c|c|}
\hline Constructs & CA & No. of items \\
\hline Expectations & 9.64 & 22 \\
\hline Perceptions & 9.42 & 22 \\
\hline Expectations and perceptions & 9.35 & 44 \\
\hline
\end{tabular}

Source: Prepared by the author.

Table 3 shows a Cronbach's alpha "excellent" value associated with the construct tourism services

Table 1. Codes of the measuring instrument related to the analysis of quality implied in tourism services in Riobamba.

\begin{tabular}{|c|c|c|}
\hline Dimension & Code & Tourism services quality \\
\hline \multirow{4}{*}{ Tangibles } & ET1 & Modern technology \\
\hline & ET2 & Visual attraction of each facility \\
\hline & ET3 & Personal appearance of staff \\
\hline & ET4 & Attractive tangible elements: brochures \\
\hline \multirow{5}{*}{ Reliability } & FB1 & Compliance with promised service \\
\hline & FB2 & Commitment and equity when solving problems \\
\hline & FB3 & Performance of service delivery that should be of premium quality \\
\hline & FB4 & Timely delivery of service \\
\hline & FB5 & No errors \\
\hline \multirow{4}{*}{ Responsiveness } & CR1 & Communicative personnel \\
\hline & CR2 & Promptness of personnel \\
\hline & CR3 & Helpful personnel \\
\hline & CR4 & Prepared personnel \\
\hline \multirow{4}{*}{ Assurance } & SG1 & Personnel conveys trust and confidence \\
\hline & SG2 & Tourists feel safe with their service provider \\
\hline & SG3 & Friendly personnel \\
\hline & SG4 & Well-trained personnel \\
\hline \multirow{5}{*}{ Empathy } & EM1 & Personalized service \\
\hline & EM2 & Convenient itinerary \\
\hline & EM3 & Individualized attention to customers \\
\hline & EM4 & Concern about customers' requests \\
\hline & EM5 & Intuition and high understanding of tourist's needs \\
\hline
\end{tabular}

Source: Prepared by the author. 
quality in Riobamba corresponding to expectations, obtained using SPSS statistics 23.

Table 3. Cronbach's alpha of tourism services quality in Riobamba: expectations.

\begin{tabular}{|c|c|}
\hline CA & No. of items \\
\hline 9.64 & 22 \\
\hline
\end{tabular}

Source: Prepared by the author.

Table 4 shows a Cronbach's alpha "excellent" value associated with the construct tourism services quality in Riobamba corresponding to perceptions, obtained using SPSS statistics 23.

Table 4. Cronbach's alpha of tourism services quality: perceptions.

\begin{tabular}{|c|c|}
\hline CA & No. of items \\
\hline 9.42 & 22 \\
\hline
\end{tabular}

Source: Prepared by the author.

The Cronbach's alpha values obtained from the dimensions corresponding to variable tourism services quality in Riobamba regarding perceptions are presented. Thus, the values achieved using SPSS statistics 23 , which are "acceptable" and "good", are shown in Table 5.

Table 5. Cronbach's alpha of tourism services quality: perceptions per dimension.

\begin{tabular}{|l|c|c|}
\hline Dimension & CA & No. of items \\
\hline Tangibles & 7.66 & 4 \\
\hline Reliability & 8.22 & 5 \\
\hline Responsiveness & 8.12 & 4 \\
\hline Assurance & 7.86 & 4 \\
\hline Empathy & 8.72 & 5 \\
\hline
\end{tabular}

Source: Prepared by the author.

The Cronbach's alpha value obtained for the variable tourism services quality is $>0.7$, that is, it is "excellent". According to statistical theory, this result determines the reliability of the instrument. Each item of the independent variable is analyzed using measures of central tendency and dispersion, such as mean and variance. Next, correlation between the analyzed items and Cronbach's alpha is calculated after some of the items conforming tourism services quality construct had been deleted. On the basis of the theoretical model, an exploratory factor analysis is applied for the purpose of identifying underlying factors or dimensions. Regarding the correlation matrix, it is understood that the higher the determinant value, the lower the correlations within the matrix; whereas the lower the determinant value, the higher the correlations within the matrix.

That is the case for this research study where a determinant value $(D=9.367 \mathrm{E}-6)$ was obtained. On the other hand, the total explained variance (TVE) is shown along with the eigenvalues in the variances and co-variances matrix, related to the percentage proportion of the calculated variance represented in each. Thus, the total variance is expressed per component, as well as the percentage of variance associated with each factor, which is obtained by dividing the sum of the eigenvalues, which corresponds with the number of total variables.

Eigenvalues $>1$ are extracted by default and included in the analyzed matrix. A confirmatory factor analysis (CFA) was applied in this case as the study used a hypothesized model, thus the analysis was set to determine three factors or dimensions. A variance of $62.8 \%$ was determined for expectations and $55.7 \%$ for perceptions, whose service quality is calculated based on the relation expectations versus perception multiplied by the total explained variance (TEV), shown in Table 7. From this standpoint, it is possible to determine if those are the real dimensions or factors.

Table 6 shows the mean and standard deviation for each item of the independent variable "Tourism services quality", which indicates how far from the mean the values can be.

Table 8 shows Bartlett's test, where expectations and perceptions are correlated obtaining values of 0.955 and 0.848 , considered "excellent" values, and a significance level of 0.000 . As a result, the hypothesis stating that there is no correlation between the variables is rejected, and the factor analysis is justified as the Kaiser-Meyer-Olkin (KMO) test result is $>0.5$. This value is the generally accepted threshold.

\section{RECOMENDATIONS}

- Promote the analysis and application of strategies that lead to relate expectations and perceptions regarding tourism services quality, as has been done with tourist destinations in Riobamba, using statistical support and substantiation.

- Consider the dimensions analyzed in this study for future research, as well as their significant contribution to the quality of tourism services.

- Include the degree of significance that exists between expectations and perceptions of 
tourism services quality in the designing of statistical model to measure service quality in tourist destinations, as both provide reference of the supply and demand that helps to support tourist' satisfaction and local development.

\section{CONCLUSIONS}

- The validity and reliability of the instrument were calculated, obtaining excellent results (values close to one) by means of the KMO test.

- That result guarantees the adequacy of the factor analysis when evaluating both measurement instruments.

- The reliability of both measuring instruments is calculated using Cronbach's alpha (CA) statistics per dimension, obtaining excellent results. In all cases, this coefficient is $>0.7$, which indicates that the instruments are stable.
- From the results obtained using the instruments, it is noteworthy that tourists deem expectations more significant than perceptions; although they deal with responses influenced by certain criteria making it possible to conduct the satisfaction analysis.

- This research makes it possible to understand the relationship between expectations and perceptions regarding tourism services quality, which is supported by the KMO test.

- The values determined by means of Cronbach's alpha were analyzed using a factor analysis, which is used for instrument validation.

- It is demonstrated that dimensions contribute to their constructs; the reliability dimension stood out in the case of tourism services quality.

\section{REFERENCES}

Table 6. Tourism service quality: mean and standard deviation of each item.

\begin{tabular}{|c|c|c|c|}
\hline \multicolumn{4}{|c|}{ Descriptive statistics } \\
\hline Tourism services quality & Mean & Standard deviation & No. of analysis \\
\hline ET1: Modern technology & 3.57 & 0.807 & 371 \\
\hline ET2: Visual attraction of each facility & 3.67 & 0.865 & 371 \\
\hline ET3: Personal appearance of staff & 3.69 & 0.900 & 371 \\
\hline ET4: Attractive tangible elements: brochures & 3.50 & 0.954 & 371 \\
\hline FB1: Compliance with promised service & 3.57 & 0.908 & 371 \\
\hline FB2: Commitment and equity when solving problems & 3.54 & 0.973 & 371 \\
\hline FB3: Performance of service delivery that should be of premium quality & 3.45 & 1.026 & 371 \\
\hline FB4: Timely delivery of service & 3.57 & 0.962 & 371 \\
\hline FB5: No errors & 3.66 & 0.935 & 371 \\
\hline CR1: Communicative personnel & 3.59 & 0.941 & 371 \\
\hline CR2: Promptness of personnel & 3.64 & 0.885 & 371 \\
\hline CR3: Helpful personnel & 3.60 & 0.943 & 371 \\
\hline CR4: Prepared personnel & 3.71 & 0.967 & 371 \\
\hline SG1: Personnel conveys trust and confidence & 3.67 & 0.916 & 371 \\
\hline SG2: Tourists feel safe with their service provider & 3.64 & 0.977 & 371 \\
\hline SG3: Friendly personnel & 3.61 & 0.965 & 371 \\
\hline SG4: Well-trained personnel & 3.64 & 0.946 & 371 \\
\hline EM1: Personalized service & 3.56 & 0.944 & 371 \\
\hline EM2: Convenient itinerary & 3.52 & 0.976 & 371 \\
\hline EM3: Individualized attention to customers & 3.53 & 0.953 & 371 \\
\hline EM4: Concern about customers' requests & 3.56 & 0.972 & 371 \\
\hline EM5: Intuition and high understanding of tourist's needs & 3.53 & 1.043 & 371 \\
\hline
\end{tabular}

Source: Prepared by the author. 
Table 7. Service quality calculated from expectations versus perceptions using the total explained variance (TEV)

\begin{tabular}{|c|c|c|c|c|c|c|}
\hline \multirow{2}{*}{ Expectations } & \multicolumn{3}{|c|}{ Initial Eigenvalues } & \multicolumn{2}{c|}{ Extraction Sums of Squared Loadings (V) } \\
\cline { 2 - 7 } & Total & \% of variance & \% cumulative & Total & \% of variance & \% cumulative \\
\hline $\mathbf{1}$ & 12.556 & 57.072 & 57.072 & 12.556 & 57.072 & 57.072 \\
\hline $\mathbf{2}$ & 1.258 & 5.716 & 62.788 & 1.258 & 5.716 & 62.788 \\
\hline $\mathbf{2 2}$ & 0.149 & 0.679 & 100.000 & & & \multicolumn{2}{c|}{ Rotation Sums of Squared Loadings } \\
\hline \multirow{2}{*}{ Perceptions } & \multicolumn{7}{|c|}{ Eigenvalues } & \multicolumn{2}{|c|}{ Total } & \% of variance & \% cumulative \\
\cline { 2 - 7 } & Total & \% of variance & \% cumulative & 4.570 & 20.773 & 20.773 \\
\hline $\mathbf{1}$ & 9.935 & 45.159 & 45.159 & 3.876 & 17.617 & 38.390 \\
\hline $\mathbf{2}$ & 1.221 & 5.550 & 50.709 & 3.798 & 17.263 & 55.652 \\
\hline $\mathbf{3}$ & 1.087 & 4.943 & 55.652 & & & \\
\hline
\end{tabular}

Source: Prepared by the author.

Table 8. Tourism services quality construct: KMO and Bartlett's Test

\begin{tabular}{|c|c|c|}
\hline \multicolumn{2}{|c|}{ KMO and Bartlett's Test: Expectations } \\
\hline \multicolumn{2}{|c|}{ Kaise-Meyer-Olkin Measure of Sampling Adequacy } & 6.955 \\
\hline \multirow{3}{*}{ Bartlett's Test of Sphericity } & Approx. Chi-Square & 231 \\
\cline { 2 - 3 } & Degrees of freedom & 0.000 \\
\cline { 2 - 3 } & Significance & 0.491 \\
\hline \multicolumn{2}{|c|}{ KMO and Bartlett's Test: Perceptions } \\
\hline Kaise-Meyer-Olkin Measure of Sampling Adequacy & 4189.418 \\
\hline \multirow{2}{*}{ Bartlett's Test of Sphericity } & Approx. Chi-Square & 231 \\
\cline { 2 - 3 } & Degrees of freedom & 0.000 \\
\cline { 2 - 3 }
\end{tabular}

Source: Prepared by the author.

[1] Cantú, H. (2006). Desarrollo de una cultura de calidad (3 ed.). Mexico D. F., Mexico: McGrawHill.

[2] Castelluci, D. (2009). La calidad y los servicios en destinos turísticos maduros. Aportes $y$ Transferencias, 13(2), 29-44. Retrieved from http://www.redalyc.org/pdf/276/27621943003. pdf

[3] Colín, L. (2002). Las normas ISO 9000:2000 de Sistemas de Gestión de la Calidad. Boletín IIE, 26(4), 182-188. Retrieved from https://www. ineel.mx/bollSO02/tecni2.pdf

[4] Cronin, J. \& Taylor, S. (1992). Measuring Service Quality: A Reexamination and Extension. Journal of Marketing, 56(3), 55-68.

[5] Denton, K. (1991). Calidad en el servicio a los clientes. Texas, United States: Díaz de Santos.

[6] Frías, D. (2014). Apuntes de SPSS: consistencia interna. Retrieved from https://es.scribd.com/ document/355502616/ApuntesSPSS-friasnavas-pdf
[7] Olmedo, K. (2014). Evaluación de la calidad del servicio turístico de los guías de los museos del Centro Histórico de Quito (CHQ) y propuesta de mejoras. (Undergraduate Thesis). Pontificia Universidad Católica del Ecuador, Quito. Retrieved from http://repositorio.puce.edu.ec/ handle/22000/9929

[8] Parasuraman, A., Berry, L. \& Zeithaml, V. (1988). SERVQUAL: A Multiple-Item Scale for Measuring Consumer Perceptions of Service Quality. Journal of Retailing, 64(1), 12-40.

[9] Parasuraman, A., Berry, L. \& Zeithaml, V. (1993). More on improving service quality measurement. Journal of Retailing, 69(1), 140147.

[10] Peñaloza, L., Toulouse, N. \& Visconti, L. (eds.) (2007). Marketing Management: A Cultural Perspective. London, United Kingdom: Rotledge. 
[11] Pride, W. \& Ferrell, O. (1997). Marketing. Conceptos y estrategias. Mexico D. F., Mexico: McGraw-Hill.

[12] Romani, M. (2017). Calidad turística y buenas prácticas de servicios turísticos en Lima Metropolitana, 2016. (Master Thesis). Universidad César Vallejo, Lima. Retrieved from http://repositorio.ucv.edu.pe/ bitstream/handle/UCV/9084/Romani_NML. pdf?sequence=1\&isAllowed=y

[13] Stanton, W., Etzel, M. \& Walker, B. (2004). Fundamentos de marketing. Mexico D. F.,
México: McGraw-Hill. Retrieved from https:// mercadeo1marthasandino.files.wordpress. com/2015/02/fundamentos-de-marketingstanton-14edi.pdf

[14] Zeithaml, V., Berry, L. \& Parasuraman, A. (1988). Communication and Control Processes in the Delivery of Service Quality. Journal of Marketing, 52(2), 35-48.

[15] Zeithaml, V., Bitner, M. \& Gremler, D. (2005). Services marketing. Integrating Customer Focus Across the Firm (4 ed.). Mexico D. F., Mexico: McGraw-Hill. 